\title{
Real-Time Electron Tomography Based on GPU Computing
}

\author{
José A. Martínez ${ }^{1}$, Francisco Vázquez ${ }^{1}$, \\ Ester M. Garzón ${ }^{1}$, and José J. Fernández ${ }^{2, \star}$ \\ 1 Dpt Computer Architecture, Almería University, 04120 Almería, Spain \\ 2 National Center for Biotechnology (CSIC) Cantoblanco, 28049 Madrid, Spain
}

\begin{abstract}
Electron tomography (ET) has emerged as the leading technique for the structural analysis of unique complex biological specimens. Recently, real-time ET systems have appeared on the scene and they combine the computer-assisted image collection with the $3 \mathrm{D}$ reconstruction, and provide the users a preliminary structure of the specimen. This rough structure allows the users to easily evaluate the quality of the specimen and decide whether a more time-consuming processing and thorough analysis of the dataset is worthwhile. The aim of this work is to develop software for real-time ET systems. The principle of ET is based upon 3D reconstruction from projections. By means of tomographic reconstruction algorithms, the projection images in the tilt series can then be combined to yield the 3D structure of the specimen. The 3D structure has poor signal to noise ratio, so it is necessary an additional non linear filtering process in order to achieve enough resolution. Then, Matrix Weighted Back Projections (Matrix WBP) and Beltrami methods have been selected as reconstruction and filter procedures, respectively. First the Matrix WBP is applied to the input sinograms to obtain the three-dimensional structure and, next, Beltrami filter de-noises the image. Both methods are highly accelerated by GPU platforms. The power of GPU computing is then exploited to further improve the performance and yield reconstructions of biological datasets in seconds, it allows to integrate both methods on real time electron tomography systems.
\end{abstract}

Keywords: Electron tomography, Real time, Matrix Weighted Back-Projection, GPU, Beltrami filter.

\section{Introduction}

Electron tomography (ET) has made it possible to directly visualize the molecular architecture of organelles, cells and complex viruses and, in particular, it has been crucial for recent breakthroughs in life sciences 6103111]. It allows to automate specimen tilting, area tracking, focusing and recording of images

\footnotetext{
* Work supported by grants CSIC-PIE-200920I075, MCI-TIN2008-01117, JA-P08TIC-3518.
} 
under low electron-dose conditions in order to preserve the specimen from radiation damage. But, as a consequence, the images exhibit poor signal-to-noise ratio (SNR).

Recently, real-time ET systems have appeared on the scene [1517. These systems provide rough structure which allows the users to easily evaluate the quality of the specimen and decide whether a more time-consuming processing and also guide the users to select further target areas to be imaged [15 17/5]. Therefore, for fully exploitation of these real-time ET systems, reconstruction and noise reduction methods are necessary. These methods should fulfill a number of requisites to be suitable for integration in real-time ET systems:(1) reconstruction method should supply the enough accurate $3 \mathrm{D}$ structure and the nonlinear denoising methods should be used so that the noise is reduced while structural features are preserved and not blurred; (2) the methods should not have complicated parameters to be tuned, as these systems are not intended to run under interactive mode and (3) the methods should be fast to approach solutions in real-time.

Weighted backprojection (WBP) is the standard reconstruction method in the field 6 10]. In this work, three-dimensional reconstruction with WBP is addressed from a matrix perspective by formulating the problem as a set of sparse matrix-vector products [16. Moreover, the Beltrami flow filter has been chosen as an efficient noise reduction method based on a geometric diffusion flow approach [9, because it reduces the noise preserving the structural features at no huge computation time. Then, the combination of both methods is an appropriated approach to develop real-time electron tomography because it verifies the three requisites before mentioned.

In the last few years, new emerging platforms are shaking up the HPC scene. Graphics processing units (GPUs) offer massive parallelism and provide outstanding performance-to-cost ratio for scientific computing. The use of GPUs for general purpose applications has exceptionally increased in the last few years thanks to the availability of Application Programming Interfaces (APIs), such as Compute Unified Device Architecture (CUDA) 12, that greatly facilitate the development of applications targeted at GPUs. This work proposes the combination of Matrix WBP and Beltrami methods based on GPU computing in order to get reconstructed $3 \mathrm{D}$ structures with enough resolution. These methods fulfill the requisites to be integrable in current real-time ET systems. The GPU platforms are selected to accelerate both methods, achieving a significant reduction of the computing time.

The rest of the paper is organized as follows: Sections 2 and 3 describe the main foundations of Matrix WBP and Beltrami methods as reconstruction and denoising approaches respectively. Section 4 analyzes the keys of the parallel implementations of these methods on multiGPU architectures. Section 5 evaluates the GPU implementations experimentally. This paper ends with some conclusions and future work. 


\section{Tomographic Reconstruction Based on Matrix WBP}

Assuming single tilt axis geometry, the 3D problem can be decomposed into a set of independent two-dimensional (2D) reconstruction subproblems corresponding to the slices perpendicular to the tilt axis [5]. The $3 \mathrm{D}$ volume is obtained by stacking the $2 \mathrm{D}$ slices reconstructed from the corresponding sinogram (i.e. the set of $1 \mathrm{D}$ projections). Now we will thus focus on the $2 \mathrm{D}$ reconstruction problem. The projection process can be modeled as follows. The sinogram $\mathbf{p}$ is related to the slice $\mathbf{g}^{\star}$ by the discrete Radon Transform or projection operation:

$$
p_{i}=\sum_{j=1}^{m} A_{i, j} g_{j}^{\star} \text { with } 1 \leq i \leq n,
$$

where $n=n_{\text {tilts }} n_{\text {bins }}$ is the dimension of $\mathbf{p}, n_{\text {tilts }}$ being the number of projection angles and $n_{\text {bins }}$ the number of projection values obtained for every projection angle; $m=m_{x} m_{y}$ is the dimension of $\mathbf{g}^{\star}$, i.e. the total number of voxels in every slice, with $m_{x}$ and $m_{y}$ being the number of voxels in the $x$ and $y$ dimensions, respectively; and $A_{i, j}$ is a weighting factor representing the contribution of the voxel $j$ to the projection value $i$, and its value only depends on the geometry of the projections. The set of weighting factors defines the $n \times m$ matrix $\mathbf{A}$. This matrix is sparse, therefore, the projection operation can be defined as a sparse matrix-vector product, $\mathbf{p}=\mathbf{\mathbf { A g } ^ { \star }}{ }^{\star}$, where $\mathbf{A}$ is usually called the forward projection operator. Then, the system $\mathbf{p}=\mathbf{A} \mathbf{g}^{\star}$ must be solved to compute the unknown slice $\mathbf{g}^{\star}$. In practice, the system is ill-conditioned and a least square problem must thus be solved to compute an approximation of $\mathbf{g}^{\star}$. WBP is the standard method to solve this problem [14], which reconstructs the specimen by uniformly distributing the specimen density present in the projection images over computed backprojection rays. Formally, the backprojection operator can be defined by means of the matrix $\mathbf{B}$ as:

$$
g_{j}=\sum_{i=1}^{n} B_{j, i} p_{i} \quad 1 \leq j \leq m
$$

where $\mathbf{B}$ is the transpose of matrix $\mathbf{A}$, and when the number of tilt angles is large enough, the vector $\mathbf{g}$ is a good estimation of the slice $\mathbf{g}^{\star}$. In WBP a high-pass filter is applied to the projections before backprojection [14, whose burden is usually negligible. In the following, we assume that the projections are already weighted. Our Matrix WBP approach then reconstructs a 3D object as a set of independent SpMV products: $\mathbf{g}^{\mathbf{s}}=\mathbf{B} \mathbf{p}^{\mathbf{s}}$ with $1 \leq s \leq N_{\text {slices }}$ where $N_{\text {slices }}$ is the total number of slices in the volume. Note that the matrix B: (1) is involved in all the products, since the projections have the same geometry for all slices; and (2) is sparse and the location of nonzero coefficients (referred to as nonzeroes) exhibits some regular pattern related to its definition (i.e. $B_{j, i}=A_{i, j}$ ).

Nowadays, the memory requirements to store the sparse matrix are fulfilled in current computers and GPUs. Specific data structures have proven suited for the particular SpMV operation of WBP with GPU [16], since the regularity and the 
symmetry relationships between the nonzeroes of $\mathbf{B}$ can be exploited to reduce the memory access and thus accelerate the SpMV operations on GPUs. These symmetries led reduce the storage space of the data structure in nearly $75 \%$, as it is explained in depth in [16].

\section{Reduction Noise by Beltrami Method}

The Beltrami flow is an efficient noise reduction method based on a geometric diffusion flow approach [9]. As such, it considers images as maps that are embedded into a higher dimension, that is, a 2D image is considered as a 2-manifold embedded in $3 \mathrm{D}$, i.e. the image $I(x, y)$ is regarded as a surface $S=(x, y, I(x, y))$ in a 3D space [7. In this work, this idea has been extended to 3D, that is, a $3 \mathrm{D}$ volume $I(x, y, z)$ is considered as a 3 -manifold embedded in a $4 \mathrm{D}$ space $S=(x, y, z, I(x, y, z))$. Embedding the multidimensional image into a higher dimension allows the use of powerful differential geometry operators 9 . In that sense, the Beltrami flow is a geometric flow approach that aims at minimizing the area of the image manifold, driving the flow towards a minimal surface solution while preserving edges [9]. The Beltrami flow is formulated as follows [8]:

$$
I_{t}=\frac{1}{\sqrt{g}} \operatorname{div}\left(\frac{\nabla \mathbf{I}}{\sqrt{g}}\right)
$$

where $I_{t}=\partial I / \partial t$ denotes the derivative of the image density $I$ with respect to the time $t ; \nabla \mathbf{I}$ is the gradient vector, that is $\nabla \mathbf{I}=\left(I_{x}, I_{y}, I_{z}\right)$, being $I_{x}=\partial I / \partial x$ the derivative of $I$ with respect to $x$ (similar applies for $y$ and $z$ ); $g$ denotes the determinant of the first fundamental form of the surface, which is $g=1+|\nabla \mathbf{I}|^{2}$; Finally, div is the divergence operator. The term $\frac{1}{\sqrt{g}}$ in Eq. (3) acts as an edge indicator since it is proven to be the projection of the normal-to-the-surface to the vector representing the 4th dimension [9].

Therefore, the Beltrami flow is a selective noise filtering method that preserves structural features as minimizes diffusion at and across edges whereas it applies extensive diffusion elsewhere [8. The implementation of the partial differential equation derived from Eq. (3) is based upon an explicit finite difference discretization [13], using an Euler forward difference approximation for $I_{t}$ and central differences to approximate the spatial derivatives. The reader is referred to that previous work [4] for an in-depth analysis of the denoising method.

The noise reduction method based on the Beltrami flow has no complicated parameters to be tuned, as the detection of the edges and estimation of their strength is performed based on $g$, which is directly computed from the gradient. Nevertheless, the method is solved in an iterative way, and hence a number of iterations have to be specified. In a previous work it was shown that a number of iterations around 100 yielded, in general, good denoising results in ET [4].

Fig. 1 is intended to illustrate the performance of this method in terms of noise reduction and feature preservation over a representative ET dataset that was taken from the Electron Microscopy Data Bank (http://emdatabank.org). 


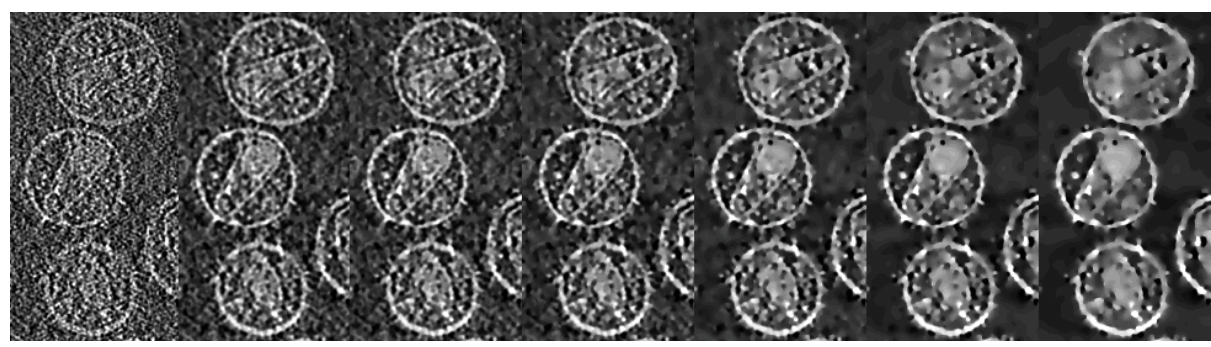

Fig. 1. From left to right, the original HIV-1 reconstruction and the results with the noise reduced at 10, 25, 50, 100, 150 and 200 iterations are shown. Only a representative slice of the $3 \mathrm{D}$ reconstruction is presented.

The dataset, whose accession code was emd-1155, is a 3D reconstruction of human immunodeficiency virions (strain HIV-1) [2]. It is clearly observed how the noise is progressively reduced with the iterations. These results show that a number of iterations of 100 are appropriate in terms of noise reduction and preservation of features.

\section{Matrix WBP-Beltrami on MultiGPU}

The GPU architecture is an accelerator of CPU computation and the input/ output data involved in every kernel executed on GPU are communicated between the main memory of CPU and the device memory of GPU. From the programmer's point of view, every GPU card is considered as a set of SIMT (Single Instruction, Multiple Threads) multiprocessors. Each kernel (parallel code) is executed as a batch of threads organized as a grid of thread blocks. In order to optimize the exploitation of the NVIDIA GPU architecture the programmer has to attend to maximize: (1) the multiprocessor occupancy, that is the ratio between the number of active warps per multiprocessor and the maximum number of possible active warps; and (2) the bandwidth memory, the memory management can be optimized if the access pattern of the different threads belonging to every half-warp (16 threads) verifies the coalescence and alignment conditions.

Both methods previously described have been implemented taking account the keys about the exploitation of the multi-GPU architecture. Two levels of parallelism have been applied in the multi-GPU implementations of WBP-Beltrami method: (1) at high level the volume is distributed among $P$ GPU cards, then every GPU computes WBP-Beltrami method on the corresponding sub-volume stored on its local device memory, consequently, communications processes between GPUs are necessary to solve data dependencies, these communications are set by means of the CPU memory; and (2) at more low level every sub-volume is computed exploiting massive parallelism of every GPU card, thus, hundreds of threads collaborate to compute WBP-Beltrami method on the sub-volume stored in the local device memory.

Matrix WBP has been formulated as a set of specific sparse matrix-vector products without data dependence among them. The sinograms are distributed 
Table 1. Run-times (s) of sequential code on a core of CPU based on 2 Quad Core Intel Xeon 2,26Ghz and GPU code on one and two Tesla C1060 cards

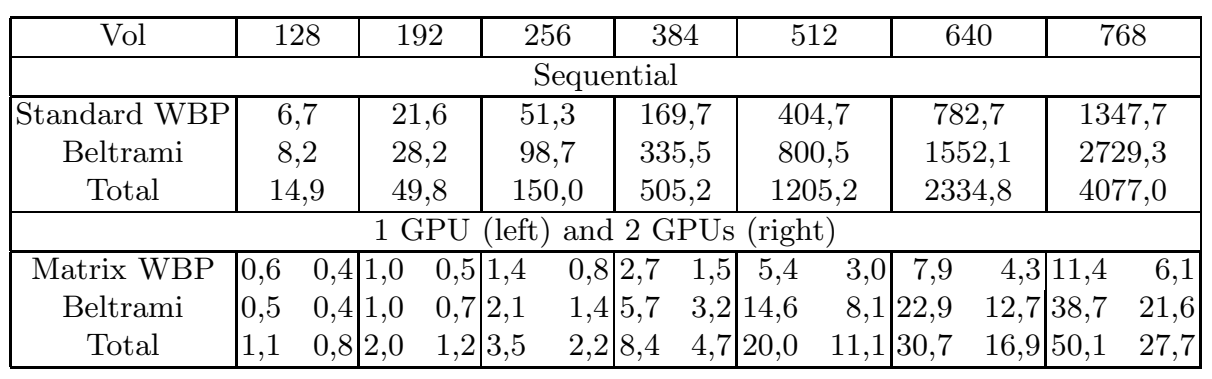

among the $P$ GPU cards. The matrix $B$ is computed by every card, aligned in the device memory and so the coalesced memory access is achieved to read the nonzeroes of $B$. Every reconstructed slice is related to one product accelerated by one GPU, so $P$ cards can simultaneously accelerate the reconstruction of $P$ slices, and the reconstructed volume is distributed among the $P$ cards. Next, every GPU sends its volume to CPU main memory and the float data format is changed to integer format and it is also distributed among the GPU cards with an additional slice in the boundaries between two sub-volumes (called halo) in order to solve the data dependence of Beltrami method to denoise the corresponding sub-volume. Then, every Beltrami iteration is accelerated by $P$ cards, when it finishes the halo slices are communicated among GPUs by means CPU.

\section{Experimental Evaluation}

The Matrix WBP-Beltrami approach was implemented with CUDA and evaluated on one core of CPU based on 2 Quad Core Intel Xeon E5520 2,26Ghz with 24GB SDRAM DDR3 1333Mhz ECC, under Linux, and an architecture of two GPUs Tesla C1060 with 4GB GDDR3 and 30 multiprocessors of 8 cores (i.e. 240 cores) at 1.2 $\mathrm{GHz}$. Synthetic datasets were created to carry out an extensive performance evaluation. They comprised a number of aligned projection images to yield cubic 3D reconstructions. The datasets had different image sizes (128, 192, 256, 512, 640 and 768 ) and number of tilt angles 180 . In the general implementation, the memory demands rapidly increase with the problem size, up to $2 \mathrm{~GB}$ in the largest test case. This amount does not turn out to be a problem in modern GPUs.

The datasets were subjected to tomographic reconstruction with the standard WBP, based on recomputation of the coefficients following by the Beltrami filter on the CPU (Standard WBP-Beltrami). The computation times of every phase of Standard WBP-Beltrami are summarized in Table 1 and are taken as reference to evaluate the speed-up achieved by the GPUs. Moreover, Table 1 includes the run times of the Matrix WBP code on one and two GPUs Tesla C1060. The run-times values shown on Table 1 highlight that: (1) The computational load of the tomographic reconstructions based on WBP-Beltrami method strongly increases when the dimensions of volume increases because the sequential times 


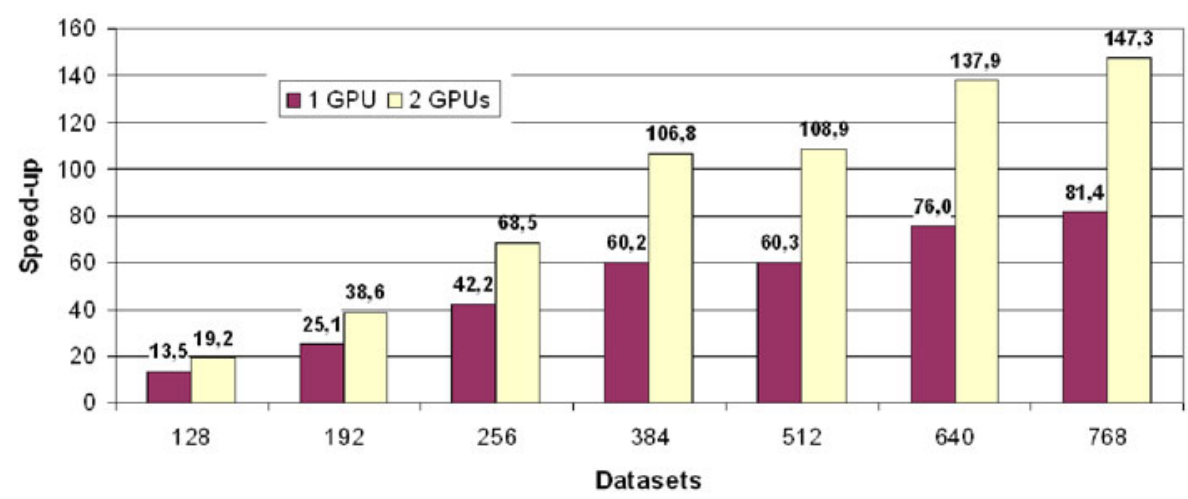

Fig. 2. (a) Speed-up factors of Matrix WBP-Beltrami implementation on one and two GPUs vs. Standard WBP-Beltrami on the test CPU

of larger test volumes are very long; (2) the GPU architecture relevantly reduces the run-times since the run times on one GPU card is reduced by two magnitude orders and (3) the GPU implementation scales on two cards due to the reduction of run-times by even half when the volume is distributed between the two cards. Fig. 2 shows that the acceleration factor thanks to exploitation of the GPU architecture is in the range $[13.5 \mathrm{x}, 81.4 \mathrm{x}]$ on one GPU card and in the range $[19.2 \mathrm{x}, 147.3 \mathrm{x}]$ on two cards, regardless of the problem size.

These results demonstrate that Matrix WBP-Beltrami based on GPU computing relevantly reduces the computing time required for tomographic. This acceleration factors lead to the capability of computing reconstructions and filter of 0.5 -1.5 GB in size in around 11-27 s, as Table 1 shows. Therefore the tomography based on Matrix WBP-Beltrami on GPUs can be joined to a real-time ET system which helps the users to select the samples with relevant information.

\section{Conclusions and Future Work}

This work has accelerated the tomographic reconstruction problem by means of GPU computing. Two methods have been selected to solve the reconstruction and denoising problems: Matrix WBP and Beltrami filter. Both methods have the appropriated characteristics to join to real-time tomographic systems, that is, the images supplied have enough resolution, they do not include complicated parameters to be tuned and their computational requirements are available on the current GPU platforms. The evaluation results have shown that both methods exploit the multi-GPU architectures achieving high acceleration factor over the run-times on CPU. Therefore these methods could be easily integrated in current real-time electron tomography systems. Consequently, the approach described in this work is expected to be of invaluable help for scientists to assess the quality of their datasets acquired during their ET sessions, and also to use this information as a guide for subsequent data collection. 


\section{References}

1. Al-Amoudi, A., Diez, D.C., Betts, M.J., Frangakis, A.S.: The molecular architecture of cadherins in native epidermal desmosomes. Nature 450, 832-837 (2007)

2. Briggs, J., Grunewald, K., Glass, B., Forster, F., Krausslich, H., Fuller, S.: The mechanism of HIV-1 core assembly: Insights from 3D reconstructions of authentic virions. Structure 14, 15-20 (2006)

3. Cyrklaff, M., Risco, C., Fernández, J.J., Jimenez, M.V., Esteban, M., Baumeister, W., Carrascosa, J.L.: Cryo-electron tomography of vaccinia virus. Proc. Natl. Acad. Sci. USA 102, 2772-2777 (2005)

4. Fernandez, J.J.: Tomobflow: Feature-preserving noise filtering for electron tomography. BMC Bioinformatics 10, 178 (2009)

5. Fernández, J.J.: High performance computing in structural determination by electron cryomicroscopy. J. Struct. Biol. 164, 1-6 (2008)

6. Fernández, J.J., Sorzano, C.O.S., Marabini, R., Carazo, J.M.: Image processing and $3 \mathrm{D}$ reconstruction in electron microscopy. IEEE Signal Process. Mag. 23(3), 84-94 (2006)

7. Fernández, J., Martínez, J.: Three-dimensional feature-preserving noise reduction for real-time electron tomography. Digital Signal Processing 20(4), 1162-1172 (2010)

8. Kimmel, R., Malladi, R., Sochen, N.A.: Images as embedded maps and minimal surfaces: Movies, color, texture, and volumetric medical images. Int. J. Comput. Vis. 39, 111-129 (2000)

9. Kimmel, R., Sochen, N.A., Malladi, R.: From high energy physics to low level vision. In: ter Haar Romeny, B.M., Florack, L.M.J., Viergever, M.A. (eds.) Scale-Space 1997. LNCS, vol. 1252, pp. 236-247. Springer, Heidelberg (1997)

10. Leis, A.P., Beck, M., Gruska, M., Best, C., Hegerl, R., Baumeister, W., Leis, J.W.: Cryo-electron tomography of biological specimens. IEEE Signal Process. Mag. 23(3), 95-103 (2006)

11. Nicastro, D., Schwartz, C., Pierson, J., Gaudette, R., Porter, M.E., McIntosh, J.R.: The molecular architecture of axonemes revealed by cryoelectron tomography. Science 313, 944-948 (2006)

12. NVIDIA: CUDA Programming guide. Version 2.3 (August 2009)

13. Press, W.H., Flannery, B.P., Teukolsky, S.A., Vetterling, W.T.: Numerical Recipes: The Art of Scientific Computing. Cambridge University Press, Cambridge (1992)

14. Radermacher, M.: Weighted back-projection methods. In: Frank, J. (ed.) Electron Tomography: Methods for Three-Dimensional Visualization of Structures in the Cell, 2nd edn., pp. 245-273. Springer, Heidelberg (2006)

15. Schoenmakers, R.H.M., Perquin, R.A., Fliervoet, T.F., Voorhout, W., Schirmacher, H.: New software for high resolution, high throughput electron tomography. Micros. Anal. 19(4), 5-6 (2005)

16. Vazquez, F., Garzon, E.M., Fernandez, J.J.: A matrix approach to tomographic reconstruction and its implementation on gpus. J. Struct. Biol. 170(1), 146-151 (2010)

17. Zheng, S.Q., Keszthelyi, B., Branlund, E., Lyle, J.M., Braunfeld, M.B., Sedat, J.W., Agard, D.A.: UCSF tomography: An integrated software suite for real-time electron microscopic tomographic data collection, alignment, and reconstruction. J. Struct. Biol. 157, 138-147 (2007) 